\title{
Plant-based production of SARS-CoV-2 antigens for use in a subunit vaccine
}

Jordan Demone $^{1 *}$, Maryam Nourimand ${ }^{1}$, Mariam Maltseva ${ }^{2}$, Mina Nasr-Sharif ${ }^{1}$ Yannick Galipeau $^{2}$, Marc-André Langlois ${ }^{2,3,4}$, and Allyson M. MacLean ${ }^{1,4}$

*joint first authors

${ }^{4}$ corresponding authors

\section{Affiliations:}

1. Department of Biology, University of Ottawa, Ottawa ON, K1N 6N5, Canada

2. Department of Biochemistry, Microbiology and Immunology, University of Ottawa, Ottawa ON, K1H 8M5, Canada

3. University of Ottawa Centre for Infection, Immunity and Inflammation (CI3)

Keywords: COVID-19, SARS-CoV-2, RBD, subunit vaccine, Nicotiana, glycoprotein

\begin{abstract}
The COVID-19 pandemic has brought to the forefront an urgent need for the rapid development of highly efficacious vaccines, particularly in light of the ongoing emergence of multiple variants of concern. Plant-based recombinant protein platforms are emerging as cost-effective and highly scalable alternatives to conventional protein production. Viral glycoproteins, however, are historically challenging to produce in plants. Herein, we report the production of plant-expressed wild-type glycosylated SARS-CoV-2 Spike RBD (receptorbinding domain) protein that is recognized by anti-RBD antibodies and exhibits high-affinity binding to the SARS-CoV-2 receptor ACE2 (angiotensin-converting enzyme 2). Moreover, our plant-expressed RBD was readily detected by $\operatorname{IgM}$, IgA, and $\operatorname{IgG}$ antibodies from naturally infected convalescent, vaccinated, or convalescent and vaccinated individuals. We further demonstrate that RBD binding to the ACE2 receptor was efficiently neutralized by antibodies from sera of SARS-CoV-2 convalescent and partially and fully vaccinated individuals. Collectively, these findings demonstrate that recombinant RBD produced in planta exhibits suitable biochemical and antigenic features for use in a subunit vaccine platform.
\end{abstract}


In this work, we describe the development of a rapid plant-based platform for the production of recombinant SARS-CoV-2 Spike Receptor-Binding Domain (RBD), which is simple, massively scalable, cost-effective and easily adaptable to reflect rapid changes in circulating viral sequences. Most importantly, our antigen closely recapitulates the biochemical and antigenic features of RBD produced in human cells, which is an essential feature for a plant-based human vaccine antigen. While most SARS-CoV-2 vaccines focus on intramuscular delivery systems, development of a subunit nasal spray vaccine offers a complementary method to increase mucosal immunity to SARS-CoV-2 variants and provide vaccine-hesitant and needle-phobic individuals with alternative immunization options. The RBD of the viral spike is the region primarily involved in binding to the cell surface receptor of the virus, the angiotensin converting enzyme 2 (ACE2) receptor (Hoffmann et al., 2020). Most neutralizing antibodies against SARS-CoV-2 are directed to the RBD (Garcia-Beltran et al., 2021; Piccoli et al., 2020; Yang et al., 2020).

A codon-optimized RBD sequence corresponding to the Wuhan-Hu-1 isolate of SARS-CoV-2 was cloned into the pHREAC vector backbone (Peyret et al., 2019) a plant-specific expression platform that has been demonstrated to achieve among the highest levels of in vivo protein expression reported in $N$. benthamiana (Fig. 1a). The RBD sequence was flanked on the $\mathrm{N}$-terminal side by an endoplasmic reticulum targeting signal peptide derived from Nicotiana tabacum PR-1a signal peptide (amino acids 1-31), and on the C-terminal side by a tandem affinity tag consisting of an 8xHis tag and a Twin-Strep-tag (Fig. 1a). The dual affinity tags were separated by a glycine-serine linker, flanked by a thrombin cleavage site (LVPRGS) that was positioned directly upstream of the affinity tags, and an ER retention signal (KDEL) located downstream and adjacent to two tandem stop codons. Initial screening of the sequence validated expression vector demonstrated that the greatest expression was achieved using Agrobacterium tumefaciens strain AGL1 when infiltrated into five-to-six-weekold $N$. benthamiana leaves that were collected four days post-infiltration (dpi) (Fig. 1b). Margolin et al. 2020, had previously demonstrated much enhanced expression of a trimeric Spike mimetic protein in N. benthamiana when co-expressed in the presence of human calreticulin (Margolin, et al., 2020), thus we assessed expression of RBD in the presence and absence of this chaperone. Co-infiltrating with calreticulin (CRT) produced a noticeable increase in RBD expression levels (Fig. 1c) and we thus opted to co-express calreticulin with RBD in subsequent infiltrations with a consideration that the human chaperone may promote proper RBD folding. All RBD samples revealed a single band that migrated slightly above the expected molecular weight of $31.3 \mathrm{kDa}$, and slightly above a mammalian-expressed RBD control (Fig. 1d).

To scale up purification, we agroinfiltrated production batches of 25-30 five-to-six-week old plants (equivalent to approximately 200 grams fresh biomass) under a vacuum. Protein was partially purified by passing lysate through a Ni-NTA column, corresponding to purification of the 8xHis moiety of the tag. Fractions corresponding to the peak detected at $280 \mathrm{~nm}$ were visualized using immunoblot analysis to confirm 
presence and integrity of protein and were then loaded onto a StrepTrap column. Peak fractions eluting from that column were visualized via immunoblot, combined, concentrated, and digested overnight with thrombin to remove the 8xHis-Twin-Strep-tag. The digested sample was passed through the StrepTrap column a second time to remove the cleaved tag, and aliquots of the purified protein were analyzed on a Coomassie gel (Fig. 1e). A band on the Coomassie gel was visualized migrating at approximately $35 \mathrm{kDa}$, corresponding in migration to the band recognized on the RBD immunoblot by anti-His antibody. SARS-CoV-2 Spike protein has $22 \mathrm{~N}$-linked glycosylation sites, two of which are located within RBD (Zhou et al., 2021). Peptide- $N$-glycosidase F (PNGase F) digestion of the partially purified protein confirmed that the plant produced RBD protein was glycosylated (Fig.1f).

We next performed a comprehensive assessment of the function and folding of RBD produced in $N$. benthamiana. Correct folding of the RBD antigen is critical for its interaction with the human ACE2 receptor and for the development of antibodies that will target and neutralize native virus and viral variants. To evaluate plant-produced RBD folding and binding kinetics, we first examined this interaction with the human ACE2 receptor. For this purpose, binding kinetics between soluble serially titrated ACE2 protein and immobilized plant RBD were evaluated by indirect enzyme-linked immunosorbent assay (ELISA). We found that plantproduced RBD readily binds to ACE2 with high affinity $\left(K_{D}=8.21 \mathrm{nM}, \mathrm{R}^{2}=0.993\right)$, comparable to the RBD expressed in mammalian $293 \mathrm{~F}$ cells $\left(\mathrm{K}_{\mathrm{D}}=6.90 \mathrm{nM}, \mathrm{R}^{2}=0.981\right)$ (Fig. $\left.1 \mathrm{~g}\right)$.

Next, we examined whether plant-produced RBD was recognized by sera from COVID-19 convalescent, partially and fully vaccinated individuals. To this effect, sera from SARS-CoV-2 exposed individuals was added to immobilized plant RBD and detection by the three classes of antibodies induced by SARS-CoV-2 infection (IgG, IgM, and IgA) was investigated by ELISA. Here, we show that plant RBD was readily detected by IgM, IgA, and IgG antibodies from naturally infected convalescent, vaccinated or convalescent and vaccinated individuals (Fig. 1h). We reveal detection and high titers of $\operatorname{IgG}$ antibodies comparable to the mammalian produced RBD across all samples tested. We also report that $\operatorname{IgM}$ and $\operatorname{IgA}$ immunoreactivity to plant-produced RBD was generally less efficient in comparison to mammalian-derived RBD. Non-specific binding of antibodies to antigen-naïve individuals was minimal for both RBD antigens tested (Fig. 1h). Our plantexpressed RBD was recognized and bound by the conformation-dependent monoclonal $\operatorname{IgM}, \operatorname{Ig} \mathrm{A}$, and $\operatorname{IgG}$ CR3022 antibodies (Fig. 1i). This constitutes a further indirect indication that the plant RBD antigen is correctly folded and did not display significant inhibitory structural alterations or post-translation modifications. However, we did observe reduced binding of the CR3022 antibody to plant-derived RBD compared to mammalian-expressed RBD. These findings may suggest that post-translational modifications can impact antibody binding to some extent, which may also explain reduced binding of human IgM and IgA antibodies. 
Lastly, we investigated the relative efficacy of human neutralizing antibodies in blocking the immobilized plant RBD's interaction with soluble ACE2 by surrogate neutralization ELISA (Abe et al., 2020; Anderson et al., 2020). As mentioned previously, most neutralizing antibodies produced by natural exposure or vaccine immunity are directed to RBD. Evidence of neutralization is an additional indicator of adequate protein folding and antigenic epitope accessibility. Human serum was added to immobilized plant produced RBD and then binding to soluble biotinylated ACE2 receptor was evaluated (Fig. 1j). We then measured the inhibition of the binding as a function of the serum dilution. This enabled the calculation of the half-maximal inhibitory dilution $\left(\mathrm{ID}_{50}\right)$ value. Our observations indicated that human antibodies were able to bind plant-expressed RBD and neutralize its binding to the ACE2 receptor with similar kinetics to the mammalian-expressed RBD (Fig. $1 \mathrm{k})$. In fact, we observed generally greater $\mathrm{ID}_{50}$ values for the plant-expressed RBD, thereby indicating that its interactions with the ACE2 receptor were more easily disrupted by neutralizing antibodies. Given that affinity for ACE2 is very similar between human- and plant-produced RBD, greater $\mathrm{ID}_{50}$ values indirectly indicate that plant RBD exposes larger numbers of neutralizing epitopes, which could be due to differences in posttranslational modifications such as glycosylation.

In summary, we have demonstrated that plant-produced RBD retains proper folding and functionality comparable to mammalian produced RBD as evaluated by binding to host cell receptor ACE2, recognition by the conformation-dependant monoclonal CR3022 antibody, and polyclonal antibodies from sera of SARS-CoV2- infected individuals. Although we did note a reduced ability of $\operatorname{IgM}$ and $\operatorname{IgA}$ antibodies to bind plantproduced RBD as compared mammalian-expressed RBD, comparable binding of IgGs, which have high affinity to the target antigen and are the main antibody isotype involved in neutralization, were observed. Additionally, plant RBD binding to the ACE2 receptor was efficiently neutralized by antibodies from sera of SARS-CoV-2 convalescent and partially and fully vaccinated individuals. Collectively, these findings demonstrate that recombinant RBD produced in $N$. benthamiana exhibits suitable biochemical and antigenic features for use in a subunit vaccine platform.

\section{Acknowledgements}

We are grateful for helpful discussions and advice from Rima Menassa and colleagues at Agriculture and AgriFood Canada, London Research and Development Centre, Canada, Emmanuel Margolin, Edward Rybicki and colleagues from Biopharming Research Unit, University of Cape Town, South Africa, and Dominique Michaud, Université Laval, Canada. We gratefully acknowledge the excellent support of Michelle Brazeau, University of Ottawa, in her management of our greenhouse. MM holds a Queen Elizabeth II Graduate Scholarship in Science and Technology (QEII-GSST) and YG holds a Canadian Institute of Health Research (CIHR) Frederick Banting and Charles Best graduate scholarship (CGS-M). This study was supported in part by a COVID-19 Rapid 
Response grant by the Canadian Institute of Health Research (CIHR, \#VR2 - 172722) and by a grant supplement by the Canadian Immunity Task Force (CITF) to MAL and funding from the University of Ottawa Faculty of Science to support greenhouse management. MAL holds a Canada Research Chair in Molecular Virology and Intrinsic Immunity.

\section{Conflict of Interest}

The authors declare no conflicts of interest.

\section{Author Contributions}

AMM and MAL conceived and supervised the project. JD, MN, MNS optimized agroinfiltration and JD \& MN optimized the purification protocol. JD performed the PNGaseF assay. MM and YG conducted in vitro functional assays and YG provided RBD control protein purified from HEK cells. JD, MN, MM, MAL and AMM contributed to the writing of the manuscript. All authors reviewed and approved the manuscript.

\section{References}

Abe, K.T., Li, Z., Samson, R., Samavarchi-Tehrani, P., Valcourt, E.J., Wood, H., et al. (2020) A simple proteinbased surrogate neutralization assay for SARS-CoV-2. JCI Insight, $\mathbf{5}$.

Anderson, D.E., Tan, C.W., Chia, W.N., Young, B.E., Linster, M., Low, J.G.H., et al. (2020) Lack of crossneutralization by SARS patient sera towards SARS-CoV-2. Emerging Microbes and Infections, 9.

Garcia-Beltran, W.F., Lam, E.C., Astudillo, M.G., Yang, D., Miller, T.E., Feldman, J., et al. (2021) COVID-19neutralizing antibodies predict disease severity and survival. Cell.

Hoffmann, M., Kleine-Weber, H., Schroeder, S., Krüger, N., Herrler, T., Erichsen, S., et al. (2020) SARS-CoV-2 Cell Entry Depends on ACE2 and TMPRSS2 and Is Blocked by a Clinically Proven Protease Inhibitor. Cell, 181.

Margolin, E.; Verbeek, M.; Meyers, A.,; Chapman, R.; Williamson, A.L.; Rybicki, E.P. (2020) Calreticulin coexpression supports high level production of a recombinant $S A R S-C o V-2$ spike mimetic in Nicotiana benthamiana. bioRxiv.

Peyret, H., Brown, J.K.M., and Lomonossoff, G.P. (2019) Improving plant transient expression through the rational design of synthetic 5' and 3' untranslated regions. Plant Methods.

Piccoli, L., Park, Y.J., Tortorici, M.A., Czudnochowski, N., Walls, A.C., Beltramello, M., et al. (2020) Mapping Neutralizing and Immunodominant Sites on the SARS-CoV-2 Spike Receptor-Binding Domain by Structure-Guided High-Resolution Serology. Cell.

Yang, J., Wang, W., Chen, Z., Lu, S., Yang, F., Bi, Z., et al. (2020) A vaccine targeting the RBD of the $S$ protein of $S A R S-C o V-2$ induces protective immunity. Nature.

Zhou, D., Tian, X., Qi, R., Peng, C., and Zhang, W. (2021) Identification of 22 N-glycosites on spike glycoprotein of SARS-CoV-2 and accessible surface glycopeptide motifs: Implications for vaccination and antibody therapeutics. Glycobiology, 31. 


\section{METHODS}

\section{Plant materials and growth conditions}

Wild-type Nicotiana benthamiana plants were grown in a greenhouse with a $20 \mathrm{~h}$ light/4h dark photoperiod at $25^{\circ} \mathrm{C}$ for five weeks prior to infiltration. Plants were fertilized two to three times per week using Miracle-Gro (24-8-16) at a concentration of $1.1 \mathrm{~g} / \mathrm{L}$. Seeds were germinated on a weekly basis and 60 three-week-old seedlings were transplanted to individual pots once a week.

\section{Gene synthesis}

The RBD sequence was obtained from the Wuhan strain (NC_045512). The sequence designed for expression in $N$. benthamiana had a C-terminal thrombin cleavage site, 8xhis tag, a Twin-Strep-tag®, and a KDEL sequence for ER-retention. The construct was codon-optimized for $N$. benthamiana expression and was synthesized by GenScript into the pHREAC vector (Peyret et al., 2019) via BsaI sites. Lectin-binding human chaperone calreticulin (NP_004334.1) was codon-optimized for N. benthamiana and synthesized in the pHRE vector (Peyret et al., 2019).

\section{Transient expression in Nicotiana benthamiana}

Prior to infiltration, pHREAC-RBD and pHRE-calreticulin were freshly transformed into Agrobacterium tumefaciens strains AGL1 and Gv2260, respectively, via electroporation. A single colony was used to inoculate each $5 \mathrm{~mL}$ starter culture, and each culture was sub-cultured once in two litres of LB broth containing $50 \mathrm{mg} / \mathrm{L}$ kanamycin at $28^{\circ} \mathrm{C}$. The final cultures were grown to an optical density at 600nm (OD600) of 0.6 and were centrifuged and resuspended in MMA buffer (10 mM MES, $10 \mathrm{mM} \mathrm{MgCl}, 200 \mu \mathrm{M}$ acetosyringone, pH 5.6). Agrobacterium containing the pHREAC-RBD construct was resuspended to a final OD600 of 0.25 and the pHRE-construct was resuspended to a final OD600 of 0.1 . The cultures were combined in a 1:1 volume ratio and incubated at room temperature for one hour prior to infiltration. Small-scale infiltration was performed by using $1 \mathrm{~mL}$ needleless syringes to inject Agrobacterium into the abaxial side of the leaves. Large-scale infiltration was performed via vacuum infiltration using three litres of infiltration solution. Leaves were gently scored, and individual plants were inverted and placed into the vacuum chamber for 2-3 minutes at a pressure of 5 bar. The vacuum was repeatedly applied until the plant was fully infiltrated. Infiltrated leaves were marked and plants were returned to the greenhouse until collection. Twenty-five plants were typically infiltrated per large-scale infiltration, equating to approximately 200 grams of fresh leaf biomass. 


\section{Total soluble protein extraction}

Leaves co-infiltrated with pHREAC-RBD and pHRE-calreticulin were collected 4-5 dpi (days post-infiltration). Leaf spines were removed, and tissue was homogenized in liquid nitrogen with mortar and pestle. Protein extracts were prepared under native conditions. For each FPLC run: 60 grams of ground tissue were resuspended in $270 \mathrm{~mL}$ of ice-cold buffer (PBS $\left(20 \mathrm{mM} \mathrm{NaH} \mathrm{PO}_{4}, 280 \mathrm{mM} \mathrm{NaCl}, 6 \mathrm{mM} \mathrm{KCl}\right), 10 \mathrm{mM}$ imidazole, $\mathrm{pH}$ 7.4) and vortexed for five minutes. Ten microlitres of Lysonase Bioprocessing Reagent (Sigma Aldrich Canada Ltd; Etobicoke, Ontario) was added and the lysate was incubated at $4^{\circ} \mathrm{C}$ for 15 minutes while gently shaking. Lysate were vortexed again and sonicated three times for 1 minute using a Kontes micro ultrasonic cell disruptor set at 70 output. Lysate was centrifuged for 45 minutes at 20,442 $\mathrm{g}$ at $4^{\circ} \mathrm{C}$ to pellet cell debris. Supernatants were filtered using several layers of cheesecloth and subsequently filtered using Corning one liter $0.22 \mu \mathrm{M}$ polyethersulfone (PES) sterilizing low-binding filters (Corning, Incorporated; Corning, NY, USA).

\section{Purification of RBD using HisPur ${ }^{\mathrm{TM}}$ Ni-NTA Resin}

Clarified total protein extract was passed through a HisPur Ni-NTA resin column (5 mL bed volume; Cytiva, Marlborough, US), pre-equilibrated with five volumes of imidazole binding buffer (PBS, $10 \mathrm{mM}$ imidazole buffer, $\mathrm{pH}$ 7.4) using a fast protein liquid chromatography (FPLC) system (AKTA Pure; GE Healthcare systems). The column was washed with twenty bed volumes of binding buffer (PBS, $10 \mathrm{mM}$ imidazole, $\mathrm{pH}$ 7.4) at a flow rate of $5 \mathrm{~mL} /$ minute. Protein was eluted using five bed volumes of elution buffer (PBS, $500 \mathrm{mM}$ imidazole, $\mathrm{pH}$ 7.4) at a flow rate of $2 \mathrm{~mL} /$ minute. Samples were collected as $2 \mathrm{~mL}$ fractions. In initial experiments, aliquots of each fraction were visualized via immunoblotting (anti-His antibody; Cat: SAB2702218, Sigma Aldrich Canada, Oakville, Canada) to identify fractions enriched for RBD, which we found to consistently correspond to a visible peak eluting from the column in fractions 25 to 35 .

\section{Purification of RBD using Strep-Tactin Resin}

Fractions corresponding to the protein peak from the HisPur Ni-NTA resin were pooled and protein was further purified using a Strep-Tactin resin column ( $5 \mathrm{~mL}$ bed volume; Cytiva, Marlborough, US) using the AKTA Pure system. The Strep-Tactin column was pre-equilibrated with five bed volumes of binding buffer (PBS, pH 7.4) prior to sample loading, and the column was then washed with 10 bed volumes of binding buffer (PBS, pH 7.4). Protein was eluted using six bed volumes of StrepTrap elution buffer (PBS, $5 \mathrm{mM} d$-Desthiobiotin, $\mathrm{pH} 7.4)$ at a flow rate of $2 \mathrm{~mL} / \mathrm{minute}$. Samples were collected as $0.5 \mathrm{~mL}$ fractions. In initial experiments, aliquots of each fraction were immunoblotted (probed with anti-His antibody) and protein was also visualized via SDS-PAGE gels stained with Coomassie blue staining solution. We reproducibly detected RBD within fractions 22 to 33 , 
which corresponded to the visible peak eluting from the column. Peak samples were collected, pooled, and concentrated to $500 \mu \mathrm{L}$ using a Vivaspin 20 column (GE Healthcare; Mississauga, Canada).

\section{Further processing of RBD protein}

Thrombin cleavage was performed to remove the tag from purified RBD protein. Ten microlitres of thrombin $(1 \mathrm{U} / \mu \mathrm{L})$ were added to the $500 \mu \mathrm{L}$ sample of purified RBD protein and incubated at $22^{\circ} \mathrm{C}$ for 20 hours. To remove the cleaved $3 \mathrm{kDa}$ tag from the final product, the reaction was passed through a Strep-Tactin resin column ( $5 \mathrm{~mL}$ bed volume) as described above. Flowthrough fractions corresponding to the peak were collected and concentrated to $500 \mu \mathrm{L}$ using a Vivaspin 20 column (GE Healthcare, Mississauga, Canada). Protein concentration was quantified using the Bio-Rad Protein Assay and using BSA for standard curve preparation. Protein purity was analyzed using SDS-PAGE and the protein was visualized using Coomassie blue staining solution.

\section{PNGase F assay}

PNGase F, an amidase, is an enzyme used to cleave $N$-linked glycans from glycoproteins and was used to assess the glycosylation status of plant-produced RBD. Briefly, $20 \mu \mathrm{g}$ of partially purified RBD protein (following elution from Ni-NTA agarose) was incubated with denaturation buffer at $100^{\circ} \mathrm{C}$ for 10 minutes. The sample was chilled to halt the denaturation process and GlycoBuffer 2 (New England Biolabs), NP-40, and PNGase F were added to the sample. The sample was incubated at $37^{\circ} \mathrm{C}$ for one hour and analyzed on an SDS-PAGE gel. Bands were visualized using Coomassie blue staining solution.

\section{Indirect ELISA to assess ACE2 binding}

To evaluate protein folding and binding, an indirect ELISA was carried out. Plant or mammalian produced SARS-CoV-2 RBD protein were diluted in sterile 1X PBS (Multicell \#311-010-CL) to $4 \mu \mathrm{g} / \mathrm{mL}$ and coated onto a 384-well Immuno plates (Thermofisher, \#460372) (12.5 $\mu \mathrm{L} /$ well) overnight at $4^{\circ} \mathrm{C}$. Plates were washed three times with $100 \mu \mathrm{L}$ of PBS-T (PBS, 1\% Tween-20) using a BIOTEK plate washer (model ELX405) and blocked for one hour with blocking buffer (PBS-T + 3\% non-fat milk powder, w/v) while shaking (700rpm) at room temperature. Biotinylated ACE2 as produced in Abe et al., 202013 was diluted in dilution buffer (PBS-T $+1 \%$ non-fat milk powder, w/v) to $258 \mathrm{ng} / \mu \mathrm{L}$ and subsequently $1: 2$ serially diluted. Plates were washed thrice with PBS-T, followed by addition of $20 \mu \mathrm{L}$ per well of titrated soluble ACE2 and incubated for one hour while shaking at room temperature. Plates were washed thrice with PBS-T followed by the addition of $20 \mu \mathrm{L}$ per well of Streptavidin-Peroxidase polymer (Sigma \#S2438) diluted in dilution buffer to $1.25 \mathrm{ng} / \mu \mathrm{L}$. After one hour incubation while shaking, plates were washed thrice with PBS-T and $20 \mu \mathrm{L}$ of freshly prepared SuperSignal 
ELISA Pico Chemiluminescent Substrate (Thermo Scientific, \#37069) (mixed 1:1 ratio and diluted in equal volume with $\left.\mathrm{dH}_{2} \mathrm{O},(\mathrm{V}: \mathrm{V})\right)$ was added to each well. Following 5 mins of incubation on a shaker, luminescence signal (RLU) was measured with BIO-TEK Synergy Neo2 plate reader at $20 \mathrm{~ms} /$ well at a read height of 1.0 $\mathrm{mm}$. Wells filled with dilution buffer in place of ACE2 accounted for background luminescence and were subtracted from the titrated ACE2 values. Dissociation constant $\left(\mathrm{K}_{\mathrm{D}}\right)$ was determined using 4-parameter curve fitting with GraphPad Prism 9.1.2 software.

\section{Indirect ELISA to evaluate anti-SARS-CoV-2 immunoreactivity in serum samples}

Plant or mammalian produced SARS-CoV-2 RBD protein were diluted in sterile $1 \mathrm{X}$ PBS to $2 \mu \mathrm{g} / \mathrm{mL}$ and coated onto a 96-well plates (VWR \#62402-959) (50 $\mu \mathrm{L} /$ well) overnight at $4^{\circ} \mathrm{C}$. Plates were washed three times with $200 \mu \mathrm{L}$ of PBS-T and blocked for one hour with a blocking buffer (PBS-T $+3 \%$ non-fat milk powder, w/v) on a shaker at room temperature. Serum samples were diluted 1:50 in dilution buffer (PBS-T $+1 \%$ non-fat milk powder, w/v). In conjunction, titration curves of conformation-dependent monoclonal IgM (Absolute Antibody, Ab01680-15.0), IgA (Absolute Antibody, Ab01680-16.0), and IgG (Absolute Antibody, Ab01680-10.0) CR3022 antibodies were used as reference material to assess protein folding. CR3022 antibodies were diluted 1:2000, followed by 1:2 serial dilution to establish a calibration curve. After blocking, plates were washed thrice with PBS-T, and followed by addition of $100 \mu \mathrm{L}$ of the respective diluted serum samples and CR3022 antibodies. The plates were incubated for two hours on a shaker at room temperature, washed thrice with PBS-T followed by the addition of $50 \mu \mathrm{L}$ of the respective secondary-HRP antibody at specified dilutions (1:4000 secondary anti-human IgG-HRP (NRC anti-hIgG\#5-HRP fusion), anti- human 1:8000 IgA-HRP (Jackson ImmunoResearch, 109-035-011) or 1:9600 anti- human IgM-HRP (Jackson ImmunoResearch, 109-035-129)). Plates were incubated for one hour on a shaker, washed thrice with PBS-T followed by the addition with $100 \mu \mathrm{L}$ of the diluted SuperSignal ELISA Pico Chemiluminescent Substrate. Luminescence intensity was measured with BIO-TEK Synergy Neo2 plate reader for 20ms/well at a read height of $1.0 \mathrm{~mm}$. Wells filled with dilution buffer in place of serum accounted for background luminescence and were subtracted from the patient serum values.

\section{Surrogate Neutralization ELISA (snELISA) assay for evaluation of neutralization in serum samples}

The described methodology was adapted from the surrogate neutralization ELISA assay as shown in Abe et al. 2020, for the evaluation of the relative inhibition of neutralizing antibodies to RBD protein from binding to soluble ACE2. Briefly, plant or mammalian produced SARS-CoV-2 RBD protein were diluted in sterile 1X PBS to $8 \mu \mathrm{g} / \mathrm{mL}$ and coated onto a 384-well Immuno plates $(12.5 \mu \mathrm{L} /$ well $)$ overnight at $4^{\circ} \mathrm{C}$. Plates were washed three times with PBS-T and blocked for one hour while shaking. Serum samples were 1:5 serially diluted in a 
dilution buffer, applied to wells $(20 \mu \mathrm{L} /$ well $)$ and incubated for 2 hours while shaking at room temperature. Plates were washed thrice, followed by the addition of biotinylated ACE2 diluted to $0.35 \mathrm{ng} / \mu \mathrm{L}$ in a dilution buffer $(20 \mu \mathrm{L} /$ well). Plates were washed thrice with PBS-T followed by the addition of $20 \mu \mathrm{L}$ per well of Streptavidin-Peroxidase polymer diluted in dilution buffer to $1.25 \mathrm{ng} / \mu \mathrm{L}$. Following one hour incubation, plates were washed thrice with PBS-T and freshly prepared SuperSignal ELISA Pico Chemiluminescent Substrate was applied (20 $\mu \mathrm{L} /$ well). Following 5 mins of incubation while shaking, luminescence intensity was measured with BIO-TEK Synergy Neo2 plate reader for $20 \mathrm{~ms} /$ well at a read height of $1.0 \mathrm{~mm}$. Control wells were filled with a dilution buffer in place of serum followed by the addition of ACE2 to assess maximum binding signal. Relative percent inhibition was calculated as follows:

$$
\% \text { Inhibition }=\left(1-\frac{\text { average mean of serum sample }}{\text { average mean of maxium signal }}\right) \times 100
$$

Serum dilution resulting in a 50\% inhibition (half-maximal inhibitory dilution $\left(\mathrm{ID}_{50}\right)$ ) of RBD protein from binding ACE2 receptor was determined using 4-parameter fitting with GraphPad Prism 9.1.2 software.

\section{RBD Production in mammalian cells}

For comparison, a mammalian RBD was produced in HEK 293F cells and purified. Briefly, a plasmid generously provided by Dr Florian Krammer (Mount Sinai, NYC) encoding the Whuhan-Hu-1 RBD (MN908947) sequence coding for the amino acid 319-541 and fused with the N-terminal SARS-CoV-2 spike secretory signal and a C-terminal hexa-histidine tag was transfected into 293F cells cultivated in Freestyle 293 expression media (Thermo Fisher, \#12338018) at $37^{\circ} \mathrm{C}, 7 \% \mathrm{CO}_{2}$, while shaking (125rpm). A total of 600 million cells resuspended in $200 \mathrm{~mL}$ were transfected with $200 \mu \mathrm{g}$ of plasmid using ExpiFectamine (Thermo Fisher, 14525). Three days post-transfection, cell supernatant was harvested by centrifugation (4000xg for 20 mins at $4^{\circ} \mathrm{C}$ ) and filtered through a low binding $0.22 \mu \mathrm{m}$ stericup vacuum filter (Millipore Sigma, S2GPU10RE). The filtered supernatant was incubated for 2 hours at room temperature with 6ml of Ni-NTA resin (Qiagen, 30210). The column containing the mix of supernatant and resin was washed four times with a washing buffer containing $20 \mathrm{mM}$ imidazole, $300 \mathrm{mM} \mathrm{NaCl}$ and $57.5 \mathrm{mM}$ of $\mathrm{NaH}_{2} \mathrm{PO}_{4} \cdot \mathrm{H}_{2} \mathrm{O}$. The RBD protein was then eluted with three column volumes of the elution buffer containing $234 \mathrm{mM}$ of imidazole, $300 \mathrm{mM} \mathrm{NaCl}$ and $57.5 \mathrm{mM}$ of $\mathrm{NaH}_{2} \mathrm{PO}_{4} \cdot \mathrm{H}_{2} \mathrm{O}$. The eluted solution was concentrated and the buffer was replaced with PBS using a 10kDa Amicon filter (Millipore Sigma, UFC901008). RBD protein integrity was verified by SDSPAGE, aliquoted to minimize freeze-thaw cycles and stored at $-80 \circ \mathrm{C}$.

\section{Patient Samples and Collection}


Use of human samples for this study was approved by the University of Ottawa Ethics Review Board: Certificates H-04-20-5727, H-04-21-6643 and H-07-20-6009. The negative sample was taken from an individual with no history of SARS-CoV-2 infection and tested with PCR. Pooled negative samples were obtained from individuals negative for SARS-CoV-2 as tested by PCR and serology assay. Serum samples from convalescent or vaccinated patients enrolled in surveillance studies from different research studies post 2019. Samples were collected using standard phlebotomy procedures. Samples were de-identified and held at $4^{0} \mathrm{C}$ for short term handling and testing at University of Ottawa CL2+ biocontainment facility. All research was performed in accordance with current guidelines and regulations.

Figure 1. Purification and evaluation of SARS-CoV-2 Spike protein Receptor Binding Domain produced in Nicotiana benthamiana. (a) Schematic representation of genetic construct used to express SARS-CoV-2 RBD in planta. The SARS-CoV-2 sequence was expressed as a recombinant protein with a dual 8xHis and Twin-Strep II tag, interspersed with glycine-serine linkers (gold boxes). An ER-retention KDEL sequence was positioned at the C-terminus, and a Thrombin cleavage site (LVPRGS) was included for tag removal. SP, signal peptide. (b) Anti-His immunoblot of samples obtained from $N$. benthamiana 2 to 5 days post-infiltration (dpi) with RBD construct in (a). Loading control at 5 dpi reproducibly demonstrates reduced abundance of protein due to initiation of tissue necrosis at this time. (c) Co-infiltration of human calreticulin increases expression levels of RBD in N. benthamiana. Samples collected 4dpi. (d) Anti-his immunoblot of purified RBD expressed in $N$. benthamiana (lane 1) and control RBD expressed in mammalian 293F cells (lane 2). L, Bio-Rad protein ladder. (e) SDS-PAGE of RBD purified from $N$. benthamiana. (f) SDS-PAGE of plant-derived RBD treated with (+) and without (-) the amidase Peptide-N-Glycosidase F (PNGaseF), an enzyme that cleaves $N$-linked glycan chains. (g to k) A comprehensive assessment of protein function of the RBD produced in N. benthamiana as it pertains to protein folding and binding to ACE2 receptor, recognition and neutralization by antibodies in sera from SARS-CoV-2 exposed individuals. (g) Indirect ELISA demonstrating binding kinetics of soluble human ACE2 to immobilized mammalian RBD (red circle) and plant RBD (blue triangle). (h) Binding and recognition of immobilized mammalian and plant produced RBD by $\operatorname{IgG}, \operatorname{IgM}$ and $\operatorname{IgA}$ polyclonal antibodies in sera of pooled naïve (unvaccinated, uninfected individuals; $n>100$ ), pooled convalescent (pooled Conv.) ( $\mathrm{n}>100)$, convalescent and vaccinated with one dose (Conv. +1 dose), convalescent and vaccinated with two doses (Conv. +2 dose), vaccinated with one dose (1 dose), vaccinated with 2 doses (2 doses) of Pfizer (BNT162b2) and naïve (PCR negative confirmed). (i) Binding and recognition of immobilized mammalian and plant produced RBD by conformation dependent monoclonal IgG, IgM and IgA CR3022 antibodies. (j) Relative inhibition percentage of anti-SARS-CoV-2 neutralizing antibodies in blocking immobilized mammalian and 
plant produced RBD from binding soluble ACE2 by surrogate neutralization ELISA assay representative of technical triplicates or quadruplicates and presented as mean \pm standard deviation. $(\mathrm{k})$ Reciprocal half-maximal inhibitory dilution $\left(\mathrm{ID}_{50}\right)$ values from $(\mathrm{j})$ for mammalian and plant-produced RBD. 
(a)

\begin{tabular}{|c|c|c|c|}
\hline SP & $\mathrm{RBD}$ & 8xHis & StreplI \\
\hline \multicolumn{2}{|c|}{ Gly-Ser linker } & \multirow{2}{*}{\multicolumn{2}{|c|}{$\begin{array}{l}\text { LVPRGS } \\
\text { Thrombin }\end{array}$}} \\
\hline & & & \\
\hline
\end{tabular}

(b)

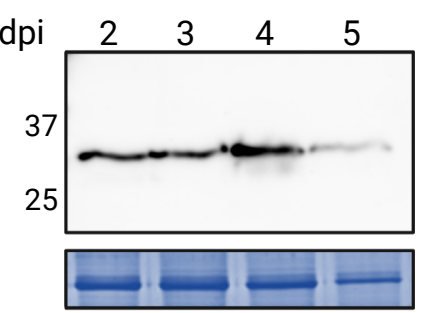

(c)

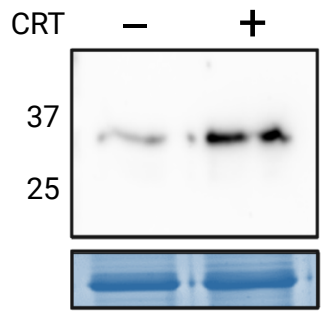

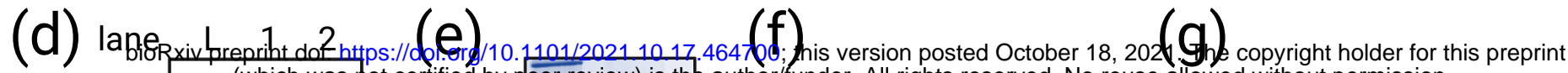
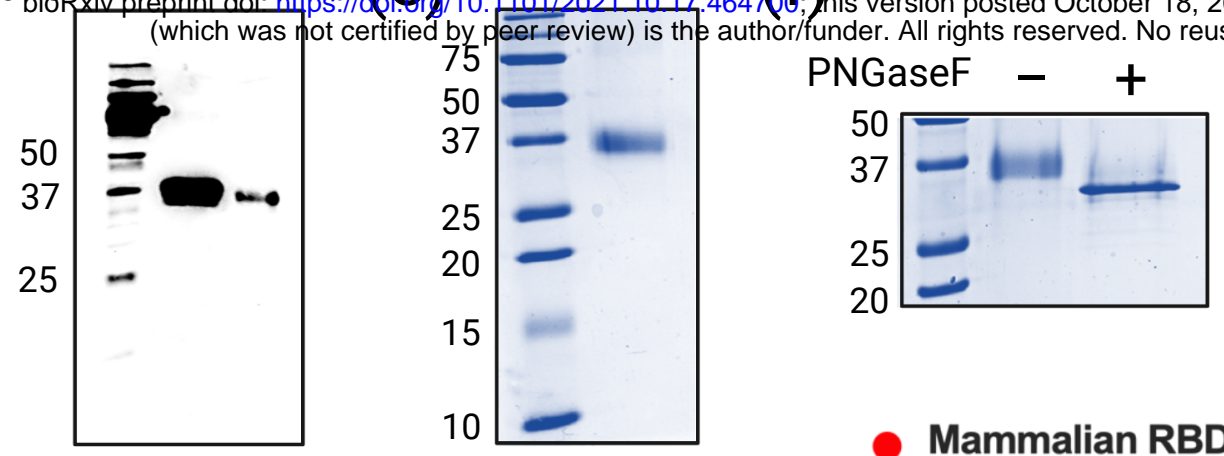

Mammalian RBD

Plant RBD

(h)

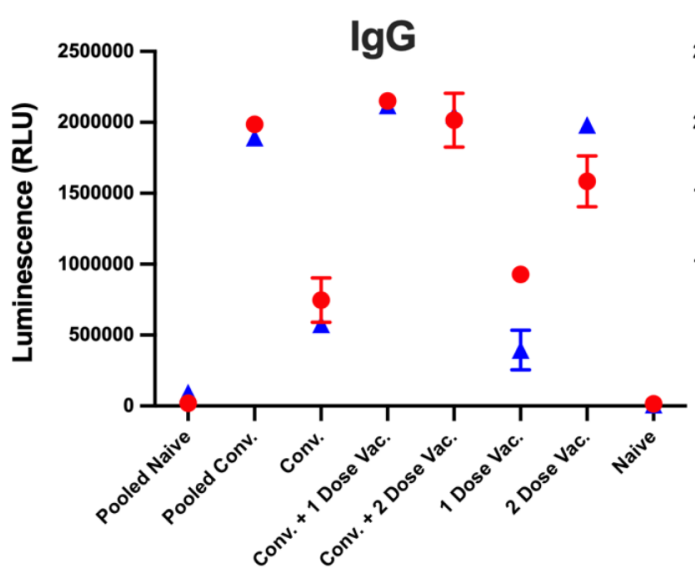

(i)

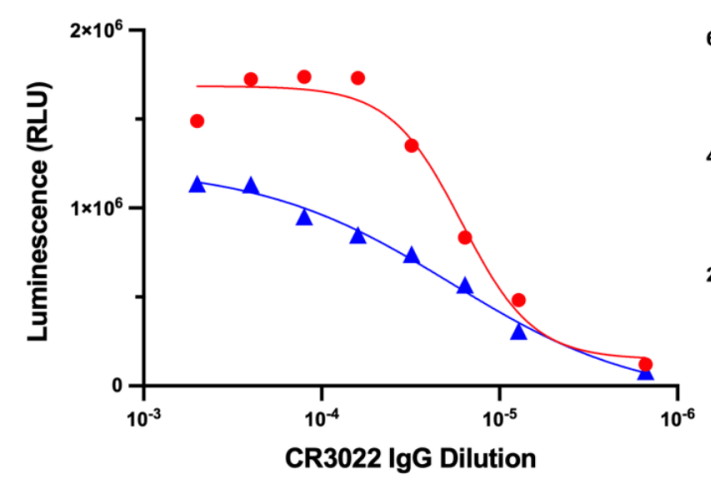

(j)

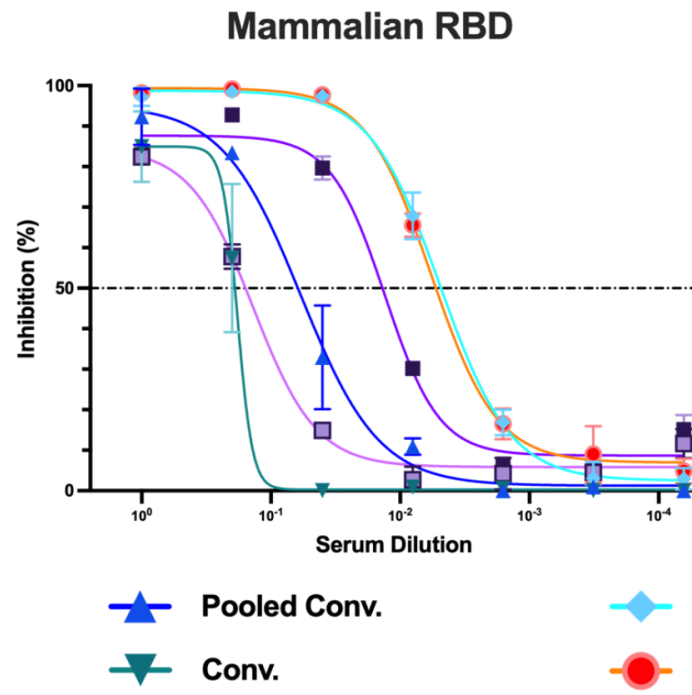

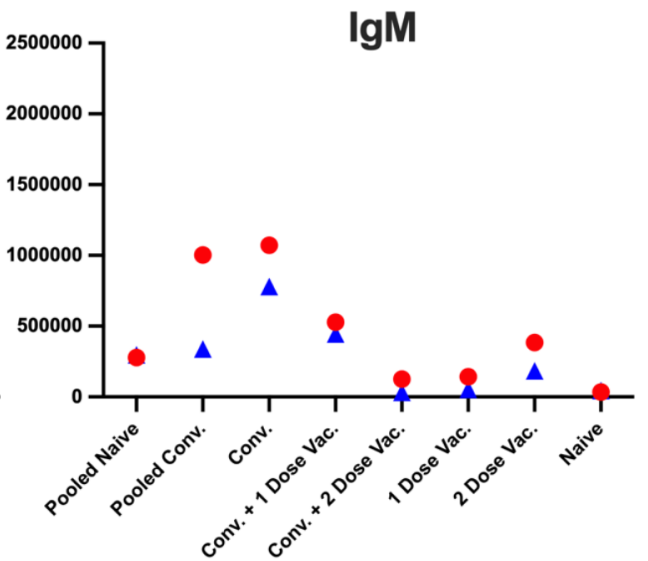

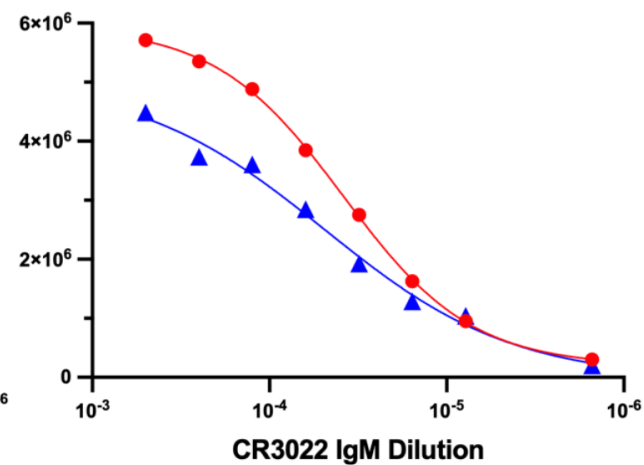

CR3022 IgM Dilution without permission.
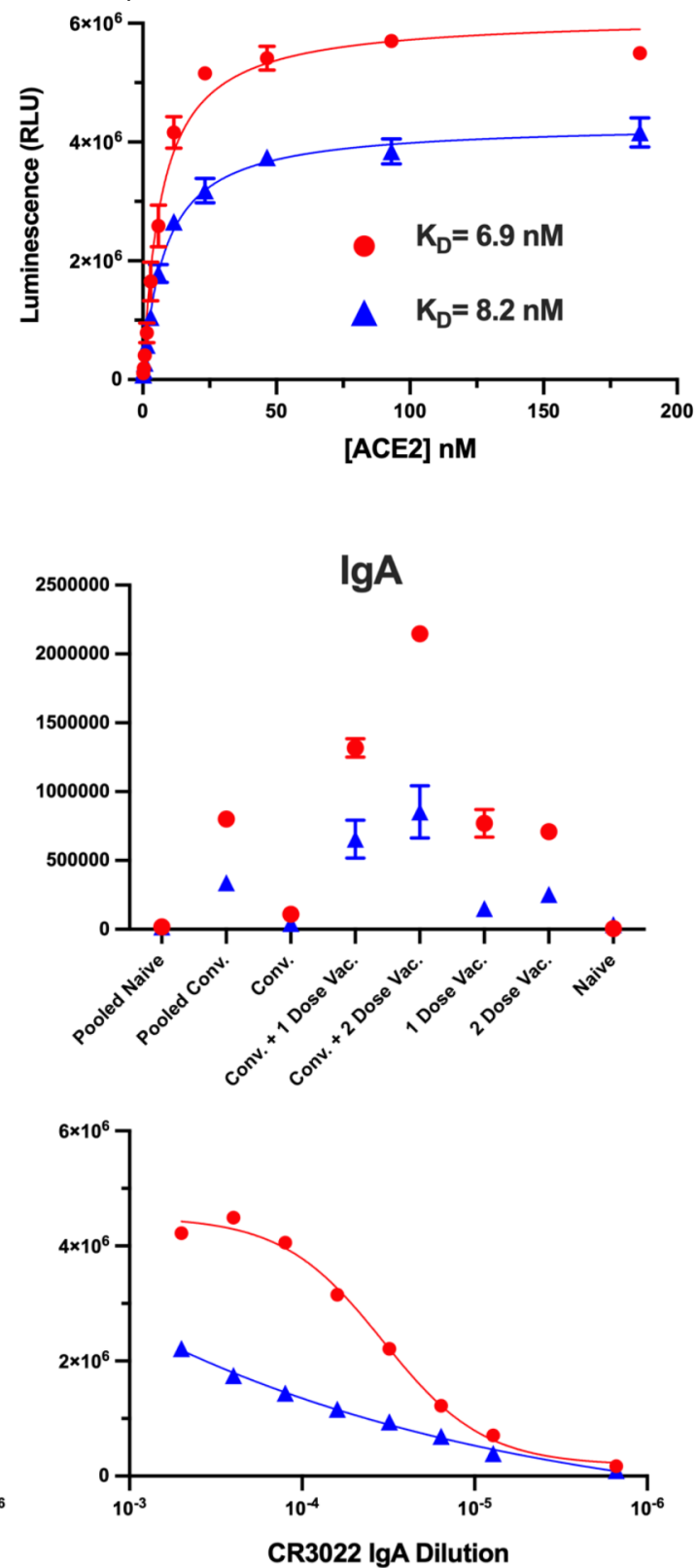

(k)

Neutralization

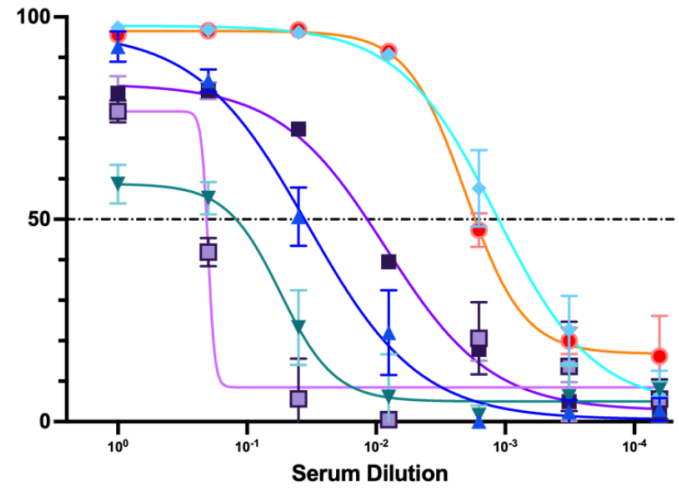

Conv. + 1 Dose

Conv. +2 Dose

Plant RBD

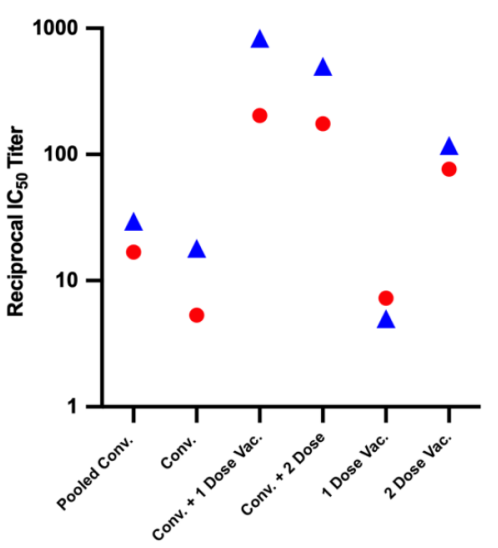

